\title{
Neue Prioritäten
}

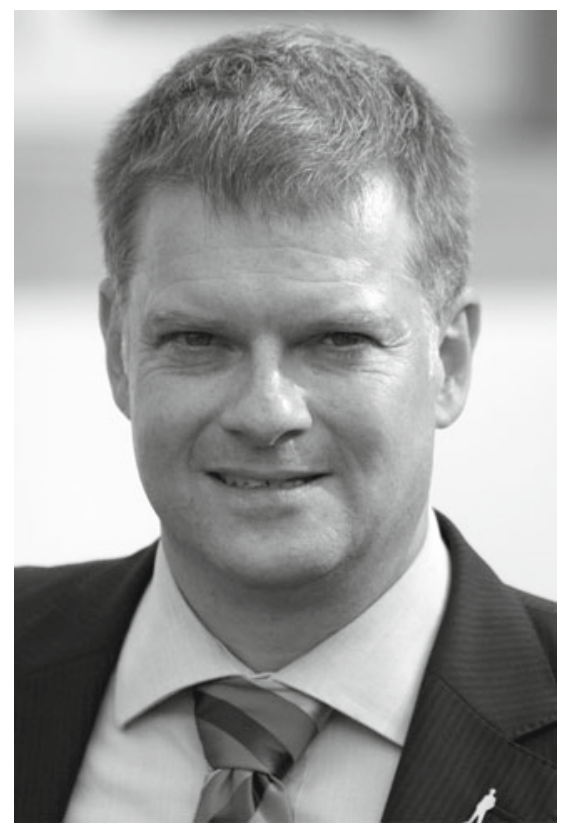

Die Kernidee von Tim Berners-Lee hinter dem Konzept des „World Wide Web“ war seine Vision global vernetzter Informationen. Aus isolierten Dokumenten sollte über direkte „Verlinkungen“ ein engmaschig verknüpftes, weltweites Informationsnetz werden. Diese Vision ist inzwischen Wirklichkeit - geradezu beispielhaft realisiert in Wikipedia, der globalen Enzyklopädie des 21. Jahrhunderts.

So revolutionär das Konzept miteinander verbundener, von jedermann abrufbarer digitaler Dokumente Mitte der 90er Jahre war - tatsächlich kennzeichnete es nur der Auftakt zu einem weit umfassenderen Paradigmenwechsel. Denn seit einem Jahrzehnt vollzieht sich - eher schleichend und nicht immer offensichtlich - die wirkliche Revolution: die dynamische Vernetzung von Programmen, Daten und Umwelt, bei der der Nutzer vom reinen Rezipienten zum Datenlieferanten geworden ist. Aus einfachen Bestellformularen wurden Shopsysteme, die sich auf Nutzerpräferenzen einstellen und ihr Angebot auf den aktuellen Standort des Nutzers (Stichwort location based services) zuschneiden - während sie zugleich Marketing, Warendisposition und Logistik steuern. Programm-Häppchen in mobilen Endgeräten werden dazu mit unterschiedlichen Datenbanken, zentralen und dezentralen Anwendungen verknüpft und greifen auf Sensoren der Endgeräte wie Mikrofone, Neigungssensoren, GPS-Empfänger, Bluetooth-Chips und Kameras zu, um mit der Umwelt des Nutzers in Kontakt zu treten.

Im Zentrum dieser schönen neuen Welt sitzen in Webseiten und Smartphones integrierte Programmschnittstellen, über die alle Datentransfers und Datenbankzugriffe gesteuert werden. Während die Angriffsfläche solcherart vernetzter Anwendungen wächst, die rund um die Uhr und von überall im Netz erreichbar sind, und zugleich Arten und Umfang der verarbeiteten (häufig personenbezogenen) Daten zunehmen, entstehen die Anwendungen („Apps“, „Web-Applikationen“) immer häufiger außerhalb der Entwicklungsabteilungen etablierter Softwärehäuser. In Werbeagenturen und bei Mediengestaltern findet man jedoch selten ein striktes Versionsmanagement, eine saubere Trennung zwischen Entwicklungs-, Test- und Produktivumgebung sowie ein strukturiertes Ticket-System. Prozessdisziplin wird vielerorts einer (falsch verstandenen) „agilen“ Softwareentwicklung geopfert.

Kein Wunder, dass seit Jahren die Standard-Fehler SQL Injection und Cross Site Scripting auf Platz 1 und 2 der OWASP Top Ten ${ }^{1}$ aufscheinen, während sich zugleich die Zahl der Web-basierten Angriffe jährlich verdoppelt. Eine Studie der Independant Oracle User Group vom Oktober 2011 legt den Finger in die Wunde: Nach ihren Maßnahmen zum Schutz vor SQL Injection-Angriffen gefragt, antworteten 34\% der Unternehmen mit "I don't know" und 30\% mit "No". Dabei betrieben 70\% der Befragten mindestens 10 und 38\% sogar mehr als 100 Datenbanken. ${ }^{2}$

Das Internet stellt nicht nur den Datenschutz vor zahlreiche neue Herausforderungen. Auch die IT-Sicherheit muss andere Prioritäten jenseits der klassischen „CIA“-Ziele (Confidentiality, Integrity, Availability) setzen - denn weder ein starkes Login noch die verschlüsselte Datenübermittlung oder ein gespiegeltes Rechenzentrum schützen vor Angreifern, die über Programmierfehler in Web-Applikationen Server oder Client-Systeme unter ihre Kontrolle bringen.

\section{Dirk Fox}

1 http://owasp.de/top10, siehe auch Gateway DuD 10/2006.

2 http://www.oracle.com/us/products/database/2011-ioug-data-security-survey-516534.pdf, Database are more at risk than ever, 2011 IOUG Data Security Survey, October 2011. 